\title{
Abnormal Neuromuscular Transmission in an Infantile Myasthenic Syndrome
}

\author{
James W. Albers, MD, PhD, John A. Faulkner, PhD, Karerina Dorovini-Zis, MD, Kate F. Barald, PhD, \\ Rachel E. Must, MS, and Richard D. Ball, MD, PhD
}

\begin{abstract}
A term infant required intubation for respiratory depression. Examination revealed hypotonia and areflexia with intact extraocular movements. Electrodiagnostic studies demonstrated defective neuromuscular transmission characterized by borderline low motor evoked amplitudes, profound decremental responses at all stimulation rates, and moderate facilitation ( 50 to $740 \%$ ) 15 seconds after 5 seconds of $50 \mathrm{~Hz}$ stimulation. Repetitive muscle action potential responses were not recorded following stimulation of nerves by single shocks. Sensory evoked responses and needle electromyographic findings were normal, as were acetylcholine receptor antibody levels. Results of muscle histochemical analyses, including acetylcholinesterase stains, were normal. End-plate histometric analyses demonstrated only a slight reduction in mean synaptic vesicle diameter compared with that in an adult control subject. In vitro muscle contractile properties, stimulating the muscle directly, were normal. Anticholinesterase medications were ineffective. Guanidine produced clinical deterioration. The amplitude of motor evoked responses progressively declined, whereas the percentage of decrement and amount of post-tetanic facilitation increased. Although the nature of the transmission defect was not identified, the data are consistent with abnormal acetylcholine resynthesis, mobilization, or storage without abnormality of release or receptors.
\end{abstract}

Albers JW, Faulkner JA, Dorovini-Zis K, Barald KF, Must RE, Ball RD: Abnormal neuromuscular transmission in an infantile myasthenic syndrome. Ann Neurol 16:28-34, 1984

Numerous neuromuscular disorders that clinically resemble myasthenia gravis have been described in neonates and infants. The physiological abnormalities identified have led to a better understanding of neuromuscular transmission and are of great interest to the clinician [19]. We report a new infantile myasthenic syndrome characterized by decremental motor responses, prominent facilitation, and normal muscle structure and contractile properties without evidence of elevated acetylcholine receptor (AChR) antibody titers.

\section{Case Report}

A $3.3 \mathrm{~kg}$ term male infant was described as hypotonic with rare spontaneous movements and was noted to have intermittent apnea. The infant was transferred to Ann Arbor at 1 week of age and required immediate intubation for respiratory depression. Examination revealed diffuse weakness and areflexia with intact extraocular movements and pupillary reflexes. Episodic increases in spontaneous extremity movement and transient improvement in respiratory variables without assisted ventilation suggested defective neuromuscular transmission. Nevertheless, repeated edrophonium and neostigmine tests were negative. Results of routine tests of serum, urine, and cerebrospinal fluid (CSF) were normal. Computed tomographic (CT) findings were normal, as was the initial electroencephalogram (EEG). Electroneuromyography at 2 weeks of age demonstrated a severe defect of neuromuscular transmission, to be described. At 3 weeks of age the infant had a ten-second episode of tonic-clonic extremity movements. Repeat CSF examinations showed no abnormalities, although a repeat $\mathrm{CT}$ scan was suggestive of a subarachnoid hemorrhage. Later this finding was interpreted as delineation of the falx by the high-resolution scanner. Repeat EEG was unchanged. Serum and stool botulinum toxin assays were negative, and $\mathrm{AChR}$ antibody titers were not elevated. Family history revealed consanguinity, the mother and father being first cousins. The patient was their only child.

Throughout the next 3 months transient improvement was noted, frequently consisting of vigorous spontaneous extremity and facial movements. Unassisted ventilation was possible for up to 4 hours, but mechanical ventilation was always reinstituted. By 3 months of age, active reflexes (fatigable) and unsustained ankle clonus supported the clinical impression of superimposed upper motor neuron involvement, presumably related to a superimposed hypoxic encephalopathy. Repeated trials of anticholinesterase medications were ineffective. Guanidine produced clinical deterioration after three days of maintenance at $18 \mathrm{mg} / \mathrm{kg} /$ day. Discontinuation resulted in subjective clinical improvement several days later. Over the next few months spontaneous improvement was less evident, and the patient died at 8 months of age of pneu-
From the Neuromuscular Section, The University of Michigan Medical Center, Ann Arbor, MI 48109.

Received Sept 17, 1982, and in revised form Sept 1 and Dec 13, 1983. Accepted for publication Dec 18, 1983.
Address reprint requests to Dr Albers, Department of Neurology, B4952, Clinical Faculty Office Bldg, The University of Michigan Medical Center, Ann Arbor, MI 48109. 

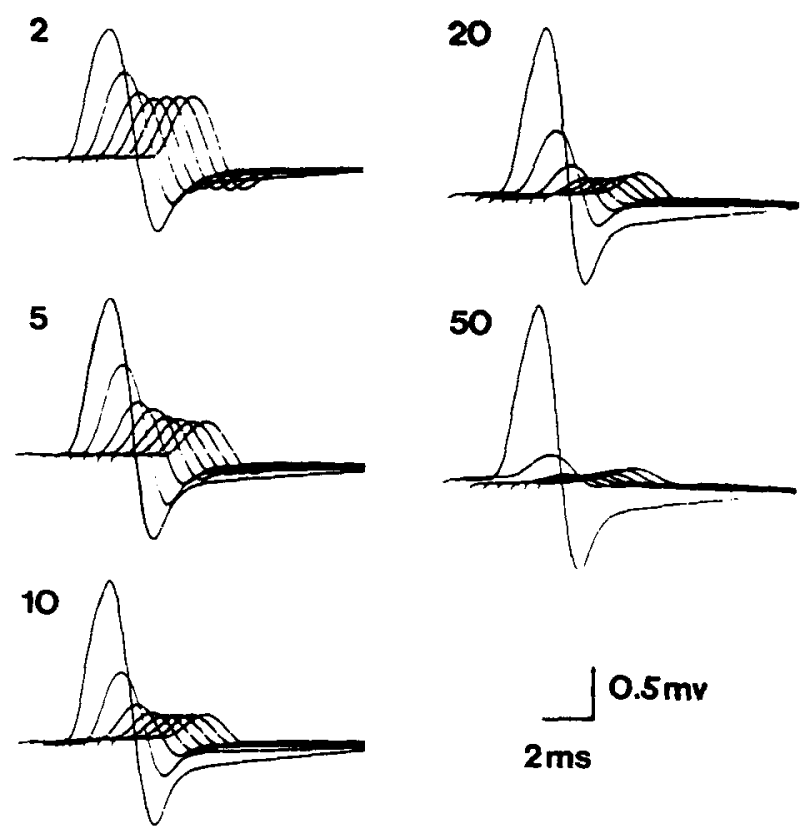

Fig 1. Ulnar compound muscle action potentials evoked by supramaximal repetitive stimulation at 2 to $50 \mathrm{~Hz}$, demonstrating a decremental response most prominent at the highest stimulation rates.

monia. Postmortem examination demonstrated a chronic sagittal sinus thrombosis with a diffuse encephalopathy.

\section{Electrodiagnostic Studies}

Sensory and motor nerve conduction studies were performed using conventional techniques of supramaximal percutaneous nerve stimulation. Sensory nerve action potentials (SNAPs) were of normal amplitude. Conduction velocity and distal latencies were normal for age. Compound muscle action potentials (CMAPs) were similarly normal, although the amplitudes were borderline low. Repetitive motor nerve stimulation recording from appropriate muscles demonstrated a profound decremental response for all nerves evaluated. The decrement was present at all rates of stimulation from 1 to $50 \mathrm{~Hz}$, with the greatest decrement at the higher rates (Fig 1). With continued stimulation there was evidence of a mild repair in the decremental response (early dip phenomenon), although there was no evidence of an incremental response. By 2 months of age moderate facilitation ( 50 to $740 \%$ ) of the initial CMAP amplitude was demonstrated 15 seconds after 5 seconds of $50 \mathrm{~Hz}$ stimulation; the decremental response to repetitive stimulation persisted (Fig 2). The post-tetanic facilitation was present in all muscles tested. SNAPs did not demonstrate a decremental response to repetitive stimulation, and repetitive motor responses were never recorded following a single nerve stimulus.

Over the subsequent 2 months SNAPs remained

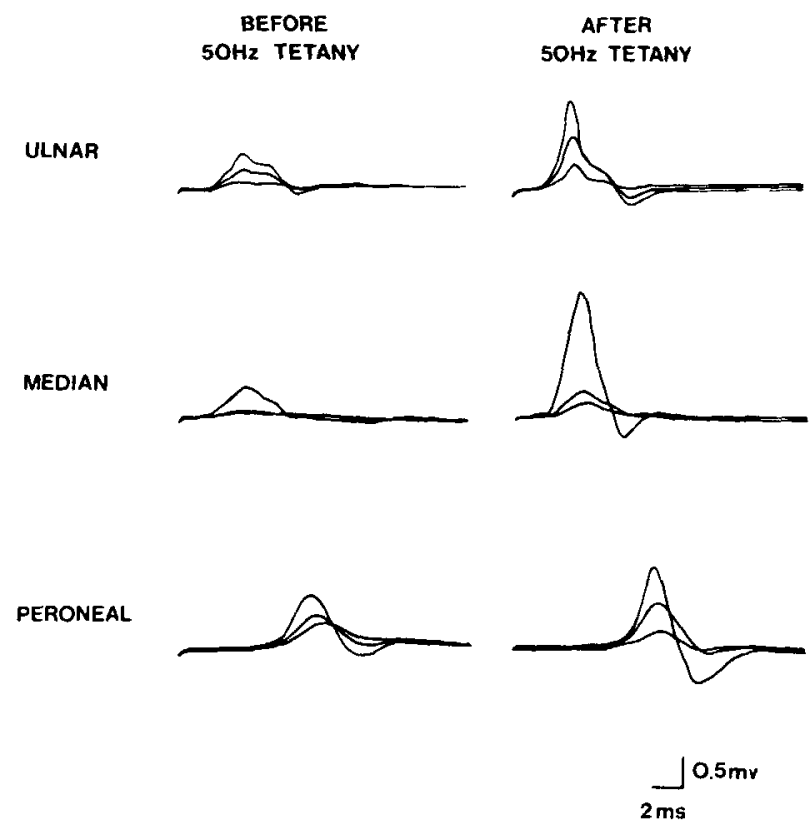

Fig 2. Ulnar, median, and peroneal compound muscle action potentials evoked by supramaximal $2 \mathrm{~Hz}$ stimulation, before and 15 seconds after 5 seconds of $50 \mathrm{~Hz}$ tetanic stimulation. Each recording represents three successive responses (initial response largest; third response smallest).

unchanged, although conduction velocities increased appropriately with increasing age. CMAPs progressively declined in amplitude, and the percentage of decrement and amount of post-tetanic facilitation increased. Intravenously administered edrophonium had no apparent effect upon either.

Concentric needle electromyography demonstrated only a paucity of motor unit action potentials (MUAPs). MUAPs that were activated by positional changes in the extremity or that occurred spontaneously appeared normal. It was never possible to demonstrate unequivocally moment-to-moment variability in individual MUAP amplitude. Abnormal spontaneous activity (fibrillation potentials or positive waves) were not observed during repeated examinations.

\section{In Vitro Studies}

All studies were performed on a biopsy specimen obtained at 3 months of age from the flexor carpi radialis muscle.

\section{Light Microscopy and Routine Histochemistry}

The only abnormality noted on examination of frozen sections stained with the routine ATPase stain at $\mathrm{pH}$ 9.4 was the presence of a few isolated small type II fibers. There was no type grouping or large group atrophy. Other fibers within the section had diameters ranging from 15 to $19 \mu$, normal for the parient's age. Modified trichrome as well as routine H\&E stains revealed no abnormalities in the muscle fiber architec- 


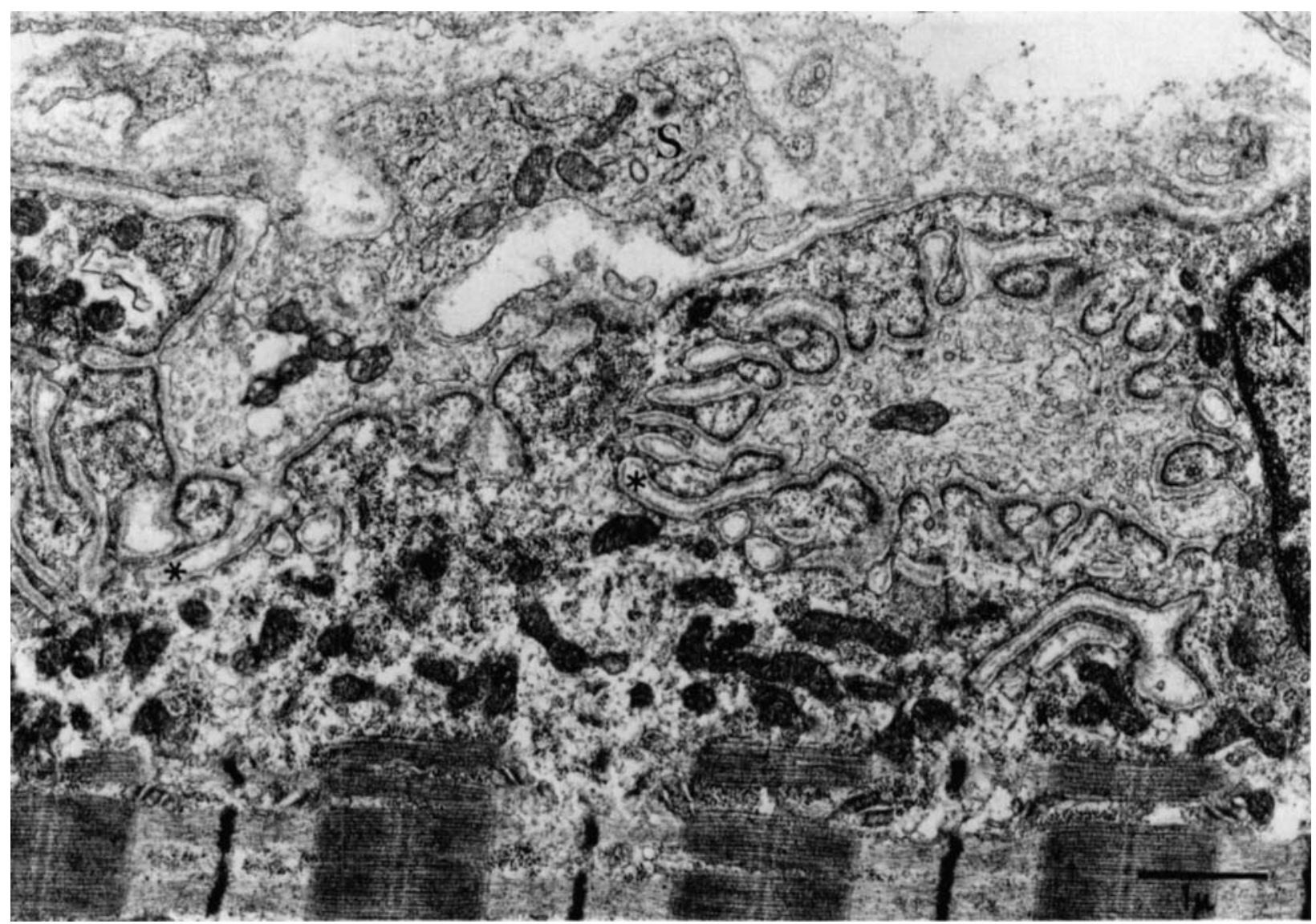

ture, a normal amount and distribution of connective tissue, and no inflammatory reaction. Nerves and muscle spindles visualized with the Goshgarian procedure [9] were within normal limits. Nonspecific esterase, cholinesterase, succinic dehydrogenase, phosphorylase, and oil red $\mathrm{O}$ stains revealed no additional abnormalities.

\section{Electron Microscopy}

The biopsy specimen was fixed in $3 \%$ phosphatebuffered glutaraldehyde and processed for electron microscopy by conventional techniques. End-plate regions were identified on $1 \mu \mathrm{m}$ thick sections stained with toluidine blue. A total of nine end plates were studied in multiple thin sections through each end plate. Thin sections were stained with uranyl acetate and lead citrate and examined in a Zeiss 109 electron microscope. All end-plate regions identified were photographed and analyzed. An image analyzer was used for histometric analyses of the nerve terminal area and the number of synaptic vesicles per unit nerve terminal area. The outer diameters of randomly selected synaptic vesicles $(\mathbf{n}=287)$ were measured at $\times 102,000$ final magnification. Muscle fibers appeared ultrastructurally normal with the exception of focal smearing of the $\mathrm{Z}$ band at the periphery of one fiber. Motor end
Fig 3. Motor end-plate region without subjective abnormality. Asterisks indicate secondary synaptic clefts. Quantitative evaluation indicated a slightly but significantly reduced mean vesicle diameter compared with that in an adult control, without differences in mean nerve terminal area or number of synaptic vesicles per unit area. $(\mathbf{N}=$ sarcoplasmic nucleus; $\mathrm{S}=$ Scbwann cell. $)$ $(\times 16,000$.

plates were well developed without apparent abnormality (Fig 3). Comparison with muscle from an adult control subject revealed no significant difference in mean nerve terminal area or number of synaptic vesicles per unit nerve terminal area. The mean vesicle diameter was slightly but significantly reduced in the patient compared with the control (means \pm SDs, 717 \pm 9 and $764 \pm 10$, respectively).

\section{End-Plate Acetylcholinesterase}

Biopsy specimens frozen in OCT compound (Miles Laboratories) were sectioned at $-20^{\circ} \mathrm{C}$ at $26 \mu$ thickness. Nerves and motor end plates were present in the biopsy material. Nonspecific esterase and AChE histochemical stains showed a dense reaction product in the region of motor end plates (Fig 4). Preincubation with esterase inhibitors blocked the reaction. 


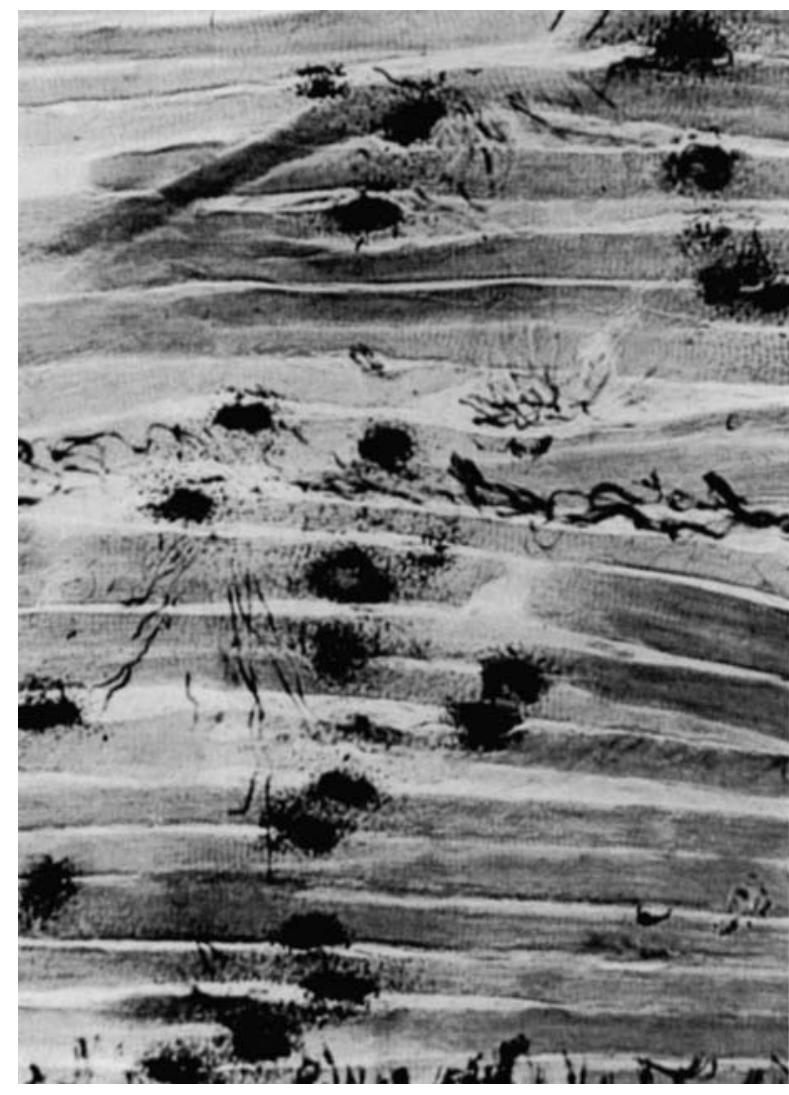

Fig 4. Acetylcholinesterase bistocbemical stain demonstrating a dense reaction product in the region of motor end plates. $(\times 1,000$ before $35 \%$ reduction.)

\section{Demonstration of End-Plate ACbR}

Frozen specimens maintained in liquid nitrogen were sectioned and placed on slides treated with $10 \mathrm{mM}$ EDTA and $0.5 \%$ gelatin. Sections were incubated for one hour with $10^{-7} \mathrm{M}$ alpha-bungarotoxin (BTX) labeled with iodine-125, $166 \mu \mathrm{Ci} / \mu \mathrm{g}$, in phosphatebuffered saline containing $1 \%$ bovine serum albumin at room temperature. Control sections were incubated in a similar concentration of unlabeled BTX to evaluate nonspecific labeling. Sections were then fixed in Bouin's fixative and counterstained with $\mathrm{H} \& \mathrm{E}$. Labeled and fixed sections were dipped in NTB 3 emulsion (Kodak), dried, and stored at $4^{\circ} \mathrm{C}$ for 1 week prior to development in Dektol (1:1) (Kodak) for 5 minutes at $20^{\circ} \mathrm{C}$

Sections were examined with a Leitz phase-fluorescence microscope and photographed. The ${ }^{125}$ I-BTXlabeled slides demonstrated that end plates contained AChRs capable of binding toxin at $10^{-7} \mathrm{M}$ (Fig 5). The grain distribution and density were subjectively normal. Incubation of the specimen in unlabeled BTX prior to labeling with iodinated toxin resulted in an absence of labeling. Because of the lack of age-matched

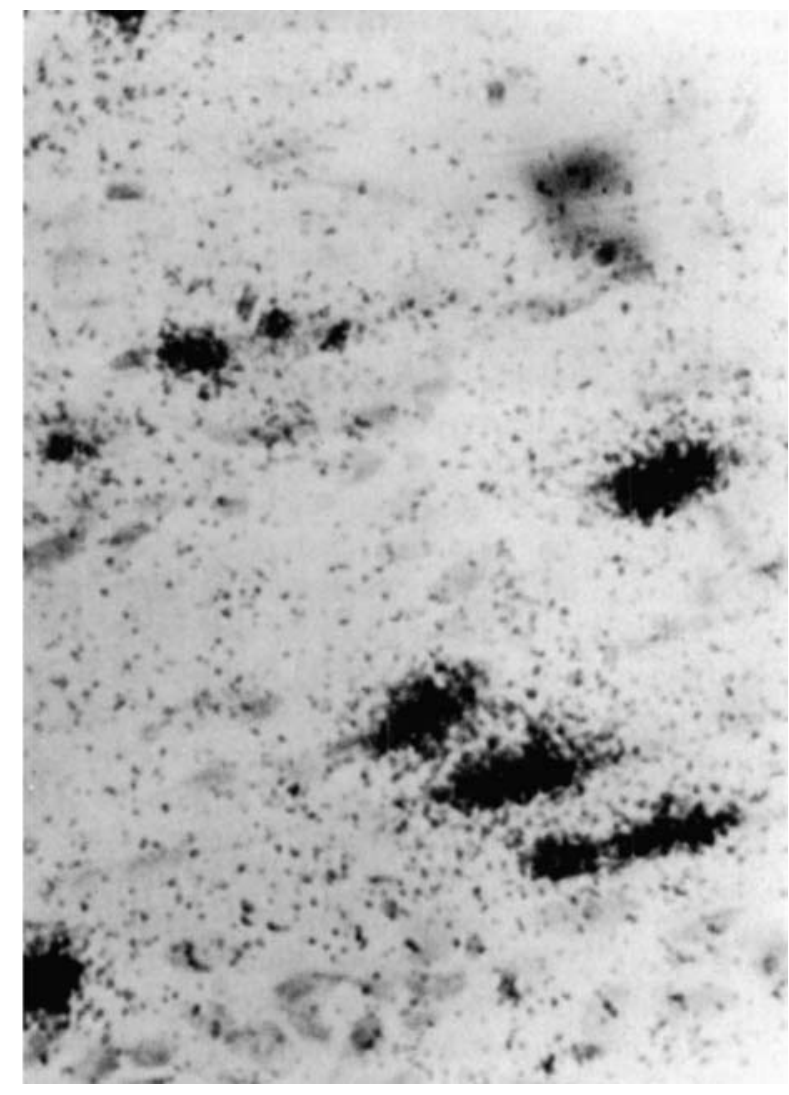

Fig 5. Autoradiogram made after incubation of muscle with ${ }^{125} I$-alpha-bungarotoxin, demonstrating that end plates contained receptors capable of binding toxin at $10^{-7} \mathrm{M}$.

controls, it was not possible to make quantitative statements regarding the number of $A C h R s$ present.

\section{In Vitro Muscle Contractile Properties}

A $600 \mathrm{mg}$ fresh specimen was placed in cooled ( 5 to $10^{\circ} \mathrm{C}$ ) oxygenated Krebs's bicarbonate saline. Samples were pinned in a dissection dish and covered with oxygenated saline at room temperature. Excitable tissue was identified with stimulating electrodes. Two bundles of excitable fibers, 18 and $22 \mathrm{~mm}$ long and approximately $30 \mathrm{mg}$ in mass, were dissected free and ligated at both ends. Both preparations were composed of segments with fibers cut at both ends. Valid measurements of contractile properties can be obtained from fiber segments [7].

Preparations were mounted between platinum stimulating electrodes in a muscle bath of $30 \mathrm{ml}$ of Krebs's bicarbonate saline with $10 \mathrm{mM}$ of glucose and $2 \mathrm{mg} / \mathrm{dl}$ tubocurarine chloride, perfused with $5 \%$ carbon dioxide in oxygen, and maintained at 36 to $37^{\circ} \mathrm{C}$. Preparations were stimulated with pulses of 40 and 100 $\mu s$ duration. Muscle bundle length was adjusted to give maximum isometric twitch tension. Stimulation rates of 
$1,10,20,50$, and $80 \mathrm{~Hz}$ were used to elicit maximum tetanic tension.

Following measurement of contractile properties, a cross-sectioned specimen was prepared and frozen in isopentane cooled to its freezing point with liquid nitrogen. Sections were incubated for myofibrillar ATPase activity at $\mathrm{pH}$ 9.4. Fiber cross-sectional areas were obtained by planimetry. On the basis of low or high myofibrillar ATPase activity, muscle fibers were classified as type I or type II, respectively. The percentages of type I and type II fibers and the mean fiber area of each type were used to calculate the percentage of the cross-sectional area occupied by each fiber type.

The maximum isometric tetanus tension ranged from 21 to $33 \mathrm{gm}$, with a twitch tension ratio of 0.21 . The range for contraction times for bundles of fibers from human skeletal muscles is 55 to $140 \mathrm{~ms}$ [3]. Because $70 \%$ of the cross-sectional area of the bundles was composed of type II fibers, the contraction time would be expected to be at the fast end of the spectrum. The experimentally obtained values of 60 and 67 $\mathrm{ms}$ for contraction time and 40 and $46 \mathrm{~ms}$ for halfrelaxation time are within the normal range for this composition of muscle fibers. Similarly, the forcefrequency relationship was not different from control values for human muscles of comparable composition. We conclude that all of the contractile properties measured were within the normal range.

\section{Discussion}

Several terms are used to describe the variety of diseases of infancy causing muscle weakness and characterized by abnormal neuromuscular transmission. The terms include myasthenia gravis, myasthenia, and myasthenic syndrome, often in association with a descriptor indicating time of onset.

Transient neonatal myasthenia gravis is restricted to infants born to mothers with myasthenia gravis [8]. The weakness is characteristic of that seen in classic myasthenia gravis but is transitory in the newborn period and presumably is related to passive transfer of maternal AChR antibody [17]. Transient neonatal myasthenia gravis develops within the first 4 days of life, and $A C h R$ antibody titers are elevated in both mother and infant $[2,14]$.

Some congenital (hereditary) myasthenic syndromes are characterized by prominent ophthalmoplegia with onset during or within 2 years of the neonatal period $[17,20]$. The impairment is not progressive, and AChR antibodies have not been detected. A decremental motor response was reported in three of five patients with congenital myasthenia, although the results of in vitro electrophysiological studies suggested that the patients represented a heterogeneous group, some having presynaptic and others postsynaptic abnormalities [20].
Familial infantile myasthenia is probably transmitted by autosomal recessive inheritance. It is often associated with respiratory depression and feeding difficulties at birth, with little or no ophthalmoplegia. Episodic weakness and apnea may be noted during the first 2 years of life, with subsequent improvement $[1,16,17]$. Involvement of siblings is common. Abnormal immune mechanisms have not been reported. A decremental motor response may be present only after exercise $[10,16]$. Electrophysiological findings are consistent with a presynaptic defect of ACh synthesis or mobilization [10].

A myasthenic syndrome characterized by generalized weakness, hyporeflexia, and refractoriness to anticholinesterase medications has been described, with ptosis beginning shortly after birth [5]. A decremental motor response was present at all stimulation rates, and a repetitive response was reported to single nerve stimulation. Several synaptic abnormalities were identified. AChE was absent from all motor end plates. The average nerve terminal size was decreased, and some end plates had abnormal postsynaptic folds. The mean store of immediately releasable quanta was reduced. Neither the repetitive motor response, nor absent AChE, nor abnormal postsynaptic folds were present in the patient we report.

Another reported congenital myasthenic syndrome was associated with ophthalmoparesis, variable extremity involvement, a decremental motor response, and repetitive muscle action potentials to single nerve stimulus [6]. The average nerve terminal size was onethird less than normal, with increased synaptic vesicle density, and there was focal degeneration of junctional folds, with loss of AChR. AChE activity was present at end plates. The ultrastructural and electrophysiological findings were consistent with a neuromuscular transmission defect secondary to changes in end-plate ultrastructure, resulting from a prolonged open time of the ACh-induced ion channel.

A familial, congenital myasthenic syndrome with a possible abnormality of $\mathrm{AChR}$ synthesis or incorporation in the postsynaptic membrane has been described in a young adult with a similarly affected sibling $[13$, 20]. One of these patients [13] had had ptosis, limb weakness, and abnormal fatigability since birth. AChR antibodies were not present, although the muscle content of AChRs was low. Results of electrodiagnostic evaluation were characteristic of those expected in acquired autoimmune myasthenia gravis.

The patient we describe is not readily classifiable into any of the categories discussed. Although he was an only child, the consanguinity suggests a congenital abnormality. The mother was asymptomatic, and neither the mother nor the infant had detectable AChR antibodies. Respiratory and extremity weakness predominated. Extraocular movements were full, making a 
clinical diagnosis of congenital (hereditary) myasthenia gravis unlikely.

The results of the electrodiagnostic studies were uncharacteristic of previously reported disorders [19]. The decremental motor response was profound ( 49 to $90 \%)$ and present at all rates $(1$ to $50 \mathrm{~Hz}$ ) of motor nerve stimulation, and there was partial repair of the decrement during continued stimulation (as opposed to a progressive decrement), consistent with the response expected in myasthenia gravis [15]. The decrement increased at higher rates of stimulation, a finding expected if the immediately available store of $\mathrm{ACh}$ was decreasing and ACh mobilization could not keep up with release. The eventual stabilization of the CMAPs with continuous stimulation represents an equilibrium between mobilization and release. A progressive decline in the CMAPs may be present in some healthy newborns, particularly at high rates of stimulation [12]. In our patient both the abnormally low CMAP amplitude and the decremental motor response were progressive and cannot be attributed to an immature neuromuscular junction. The decremental response alone could be seen in association with myotonia and was prolonged after depolarization, but myotonia was not demonstrated on needle electromyography and would not account for the reduced CMAP amplitude prior to repetitive stimulation.

Failure of repetitive motor nerve stimulation at high rates to facilitate the CMAP amplitude suggests that calcium-mediated $\mathrm{ACh}$ release was not abnormal. This finding is also consistent with both the failure of guanidine to improve neuromuscular transmission and the absence of facilitation immediately after tetanic stimulation.

Prominent facilitation of the CMAPs was observed approximately 15 seconds after brief tetanic stimulation. The time course of facilitation was unlike that reported for some patients with myasthenia gravis [18], in whom an incremental response can be demonstrated immediately after voluntary tetanus or during electrical stimulation. Tetanic stimulation increases ACh mobilization $[4,11]$. Mobilization continues for several seconds after tetanic stimulation, and the releasable store of ACh may be increased [19], perhaps explaining the substantial facilitation in CMAPs observed in our patient.

The slight but significant difference in mean vesicle diameter identified in histometric analyses of the patient end-plate region compared with that in an adult control is of unknown significance. It may reflect normal size for age, as opposed to faulty synaptic vesicle development.

The defect in neuromuscular transmission could be explained by abnormal $\mathrm{ACh}$ resynthesis, mobilization, or storage, without abnormality of $\mathrm{ACh}$ release or of AChRs. In a model in which the releasable store of
ACh was low, the end-plate potential for many muscle fibers could be below threshold for excitation of the muscle fiber action potential, and the CMAP would be reduced. Repetitive stimulation at low rates prior to mobilization would further deplete the releasable store and reduce the CMAP. Mobilization of ACh would augment the releasable store and partially repair or stabilize the decrement. Stimulation at higher rates $(20$ to $50 \mathrm{~Hz}$ ) would result in a more rapid depletion of the already reduced releasable store and a greater reduction in the CMAP amplitude. The facilitative effects of calcium would be small in such a hypothetical model, with the result being simply the release of a larger percentage of an inadequate $\mathrm{ACh}$ store. Persistent ACh mobilization several seconds after tetanic stimulation would result in "overfilling" of the releasable store [19], increased amplitude of end-plate potentials, and a short-lived increase in CMAP amplitude. In vitro microelectrode studies would be necessary to define the neuromuscular transmission defect more precisely.

Portions of this study were presented at the Annual Meeting of the American Association of Electromyography and Electrodiagnosis, Portland, OR, Oct 2, 1981, and at the Annual Meeting of the Myasthenia Gravis Association, New York, NY, Dec 4, 1981.

Many individuals participared in the evaluation and care of this patient. The authors particularly thank Drs G. W. Goldstein and S. M. Donn for their assistance.

\section{References}

1. Conomy JP, Levinsohn M, Fanaroff A: Familial infantile myasthenia gravis: a cause of sudden death in young children. J Pediatr 87:428-430, 1975

2. Donaldson JO, Penn AS, Lisak RP, et al: Antiacetylcholine receptor antibodies in neonatal myasthenia gravis. Am J Dis Child 135:222-226, 1981

3. Eberstein A, Goodgold J: Slow and fast twitch fibers in human skeletal muscle. Am J Physiol 215:535-541, 1968

4. Elmquist D, Quastel DMJ: A quantitative study of endplate potentials in isolated human muscle. J Physiol (Lond) 178:505529,1965

5. Engel AG, Lambert EH, Gomez MR: A new myasthenic syndrome with end-plate acetylcholinesterase deficiency, small nerve terminals, and reduced acetylcholine release. Ann Neurol $1: 315-330,1977$

6. Engel AG, Lambert EH, Mulder DM, et al: A newly recognized congenital myasthenic syndrome attributed to a prolonged open time of the acetylcholine-induced ion channel. Ann Neurol 11:553-569, 1982

7. Faulkner JA, Claflin DR, McCully KK, Jones DA: Contractile properties of bundles of fiber segments from skeletal muscles. Am J Physiol 243 (Cell Physiol 12):C66-C73, 1982

8. Fenichel GM: Clinical syndromes of myasthenia in infancy and childhood. Arch Neurol 35:97-103, 1978

9. Goshgarian HG: Rapid silver impregnation for central and peripheral nerve fibers in paraffin and frozen sections. Exp Neurol 57:296-301, 1977

10. Hart ZH, Sahashi K, Lambert EH, Engel AG: A congenital, familial myasthenic syndrome caused by a presynaptic defect of transmitter resynthesis or mobilization. Neurology (NY) 29:556, 1979

11. Johns RJ, Grob D, Harvey AM: Studies in neuromuscular func- 
tion. II. Effects of nerve stimulation in normal subjects and in patients with myasthenia gravis. Bull Johns Hopkins Hosp 99:125-135, 1956

12. Koenigsberger MR, Patten B, Lovelace RE: Studies of myoneural function in the full term and the premature infant. Neuropaediatrie 4:350-361, 1973

13. Lambert E: Electrophysiologic diagnosis of myasthenia gravis and other disorders of neuromuscular junction. In Tenth Neuromuscular Disease Symposium. Houston, TX, Baylor College of Medicine, 1982, p 35

14. Ohta M, Matsubara F, Hayashi K, et al: Acetylcholine receptor antibodies in infants with myasthenia gravis. Neurology (NY) 31:1019-1022, 1981

15. Ozdemir C, Young RR: The results to be expected from electri- cal testing in the diagnosis of myasthenia gravis. Ann NY Acad Sci 274:203-222, 1976

16. Robertson WC, Chun RWM, Kornguth SE: Familial infantile myasthenia. Arch Neurol 37:117-119, 1980

17. Seybold ME, Lindstrom JM: Myasthenia gravis in infancy. Neurology (NY) 31:476-480, 1981

18. Singer P, Smith L, Ziegler DK, Festoff BW: Posttetanic potentiation in a patient with myasthenia gravis. Neurology (NY) 31:1345-1347, 1981

19. Swift TR: Disorders of neuromuscular transmission other than myasthenia gravis. Muscle Nerve 4:334-353, 1981

20. Vincent A, Cull-Candy SG, Newsom-Davis J, et al: Congenital myasthenia: end-plate acetylcholine receptors and electrophysiology in five cases. Muscle Nerve 4:306-318, 1981 